\title{
Síndroma do desconforto corporal: unir o que a especialização separou
}

José M. Mendes Nunes ${ }^{1-2}$

\section{RESUMO}

O autor defende o conceito de síndroma de desconforto corporal (bodily distress syndrome) como entidade unificadora das síndromas somáticas e sintomas somatoformes cujos doentes apresentam características fisiológicas, cognitivas e comportamentais que exigem uma abordagem integrada e global. Estamos perante um conceito integrador de uma multiplicidade de pseudodiagnósticos que são efeitos colaterais da especialização médica. Operacionaliza a abordagem de doentes que, paradoxalmente, sendo dos que mais precisam de uma visão holística e integrada são os mais sectorizados e desintegrados pela abordagem das múltiplas especialidades médicas, que se limitam a adicionar rótulos que apenas contribuem para reduzir a perceção de saúde do paciente. A síndroma de desconforto corporal (SDC) assume a complexidade destes sintomas, recusa a dualidade mente-corpo e a definição pela negativa (ausência de explicação médica). Porque se trata de uma situação cuja abordagem integrada depende da relação médico-doente e da continuidade de cuidados, o médico de família é o principal responsável pela abordagem destes pacientes, sem prejuízo de episodicamente ter de recorrer a outras especialidades.

Palavras-chave: Síndromas somáticas funcionais; Sintomas somatoformes; Síndroma de desconforto corporal

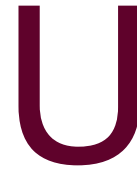
m dos problemas mais frequentes na consulta de medicina geral e familiar (MGF) são os sintomas físicos não explicados por doença orgânica ${ }^{1-5} \mathrm{ou}$, de outro modo, é a dolência sem doença.

Este tema já mereceu a atenção de um editorial ${ }^{6}$ e de uma carta ao editor ${ }^{7}$ nesta publicação. No entanto, o problema tem sido ignorado em Portugal. Muitas têm sido as designações utilizadas para mencionar estes sintomas: somatização, sintomas de somatização, sintomas físicos medicamente inexplicados (medically unexplained physical symptoms), sintomas múltiplos medicamente inexplicados (multiple medically unexplained symptoms), síndromas sintomáticas persistentes (persistent symptoms syndromes), condições baseadas em sintomas (symptom-based conditions) e síndromas somáticas funcionais (functional somatic syndromes)..$^{8-9}$

O termo "perturbação somatoforme», proposto no DSM-IV,${ }^{10}$ já referido no DSM-III, e pela CID- $10,{ }^{11}$ conotase com a origem predominantemente psicogénica, insiste na visão dualista e é de difícil aceitação pelos doentes. Para além disso, estas definições assentam na negação: os sintomas não têm uma causa física.

1. Departamento de Medicina Geral e Familiar, NOVA Medical School / Faculdade de Ciências Médicas, Universidade Nova de Lisboa.

2. USF de Carcavelos, ACeS de Cascais.
O Grupo de Trabalho dos Sintomas Somáticos (Somatic Symptom Disorders Work Group -SSDWG) ${ }^{12}$ propôs, para o DSM-V, a renomeação da categoria «Perturbações Somatoformes» do DSM-IV para «Perturbações de Sintoma Somático» (Somatic symptom disorders) por serem condições que envolvem sintomas físicos e/ou preocupações com condições médicas. O SSDWG define dois subtipos de perturbações de sintoma somático: 1) perturbação de sintoma somático complexa (Complex somatic symptom disorder); 2) perturbação de sintoma somático simples ou abreviada (Simple, or abridged, somatic syndrome disorder). ${ }^{13}$ Embora a designação «Perturbação do Sintoma Somático Complexa» incorpore a complexidade dos sintomas somáticos, como a persistência (critério A) e a associação a distorções cognitivas (critério B), existem outros aspetos que contribuem para o caráter complexo destes sintomas e que não são considerados, como o sofrimento desproporcionado e as características psicocomportamentais. Acresce ainda a incongruência da designação "Perturbação do Sintoma», parecendo que está em causa um sintoma perturbado e não uma perturbação que se manifesta por sintomas.

Alguns autores consideram o problema dos sintomas sem doença como um contínuo, cujos extremos são o normal e a somatização. ${ }^{14}$ No entanto, o DSM-IV e a CID-10 não 
têm em consideração esta continuidade, não contrariam o dualismo mente-corpo e a simplificação dos critérios pode causar uma inflação de diagnósticos, com todas as consequências de rotular a dolência como doença mental. ${ }^{15}$ Para além disso, o diagnóstico de perturbações somatoformes assenta em critérios negativos, i.e., na exclusão de doença orgânica, com duração superior a seis meses, muito pouco utilizado e de utilidade muito reduzida fora do âmbito muito especializado. ${ }^{16}$ Tendo em atenção que as definições de perturbações de somatização são demasiado restritivas, deixando de fora muitos doentes com características cognitivas, comportamentais e sociais que definem os sintomas somatoformes, Escobar e colaboradores ${ }^{17}$ defendem a classificação de somatização abreviada (abridged somatization) como forma de integrar a pulverização de síndromas funcionais resultante do artificialismo da especialização médica. Definem somatização abreviada com a presença de quatro ou seis sintomas, no homem ou na mulher, respetivamente, de uma lista de 37 sintomas somáticos não relacionados com doença. Esta é uma ideia que vem da psiquiatria, que continua a elaborar no dualismo mente-corpo e não tem, mais uma vez, em consideração a realidade e as necessidades dos cuidados de saúde primários.

Muitos outros autores ${ }^{17-19}$ entendem que as síndromas funcionais, como cólon irritável, fibromialgia, fadiga crónica e outras designações, são expressões do mesmo fenómeno e que resultaram da especialização da medicina, sendo que praticamente todas as especialidades possuem a sua síndroma funcional como consequência da sua visão afunilada (Tabela I).

Nesta sequência, alguns autores têm proposto a designação de Disorder/Syndrome of Bodily Distress, que engloba os sintomas somatoformes e as síndromas funcionais, ${ }^{20}$ e que eu traduzo como Perturbação ou Síndroma de Desconforto Corporal (SDC).

No entanto, embora os conceitos de síndroma e de perturbação sejam usados, neste contexto, de modo indistinto, a realidade é que eles são defendidos por diferentes grupos.

No processo de desenvolvimento da CID-11 surgiram dois grupos com o objetivo de elaborarem recomendações para o International Advisory Group remodelar o capítulo das perturbações mentais e comportamentais. O Primary
Care Consultation Group (PCCG) tinha o objetivo de substituir a categoria F45 (sintomas somáticos não explicados/sintomas não explicados medicamente) sobretudo para a versão da CID para os cuidados primários, a ICD-11PHC. Este grupo vem dos cuidados primários e propõe a designação de Bodily Distress (também há quem chame Stress) Syndrome. ${ }^{21}$ Outro grupo, o Expert Working Group on Somatic Distress and Dissociative Disorders (S3DWG), onde predominam peritos da psiquiatria, foi encarregado de fazer o mesmo que o PCCG mas relativamente ao capítulo de «Perturbações Somatoformes» (Somatoform disorders) e propõe a designação de Bodily Distress Disorders.

Os dois grupos fazem propostas ignorando-se mutuamente. Para o S3DWG, a Bodily Distress Disorder, em comparação com as perturbações somatoformes e neurastenia, tem as seguintes vantagens:

a) Os critérios são simplificados;

b) Sem assunções quanto à causalidade;

c) Não exige que os sintomas tenham, como critério central, o "não explicado medicamente";

d) Inclui a presença de condição física concorrente;

e) Focagem na identificação da resposta psicocomportamental positiva (excessiva preocupação pelos sintomas, medo injustificado de doença, hiperutilização dos serviços de saúde, comportamento evitante por medo de fazermal); 
f) Esta resposta surge perante qualquer sintoma (inespecífico), persistente, estressante, em um ou vários órgãos ou sistemas.

Esta entidade tem elevada concordância com as perturbações do sintoma somático da DSM-5 e baixa com a SDC de Fink e colaboradores. $^{20}$

No entanto, estes autores estudaram uma amostra de 978 doentes de neurologia, medicina interna e dos cuidados primários, com sintomas físicos não explicados por doença convencional defini-

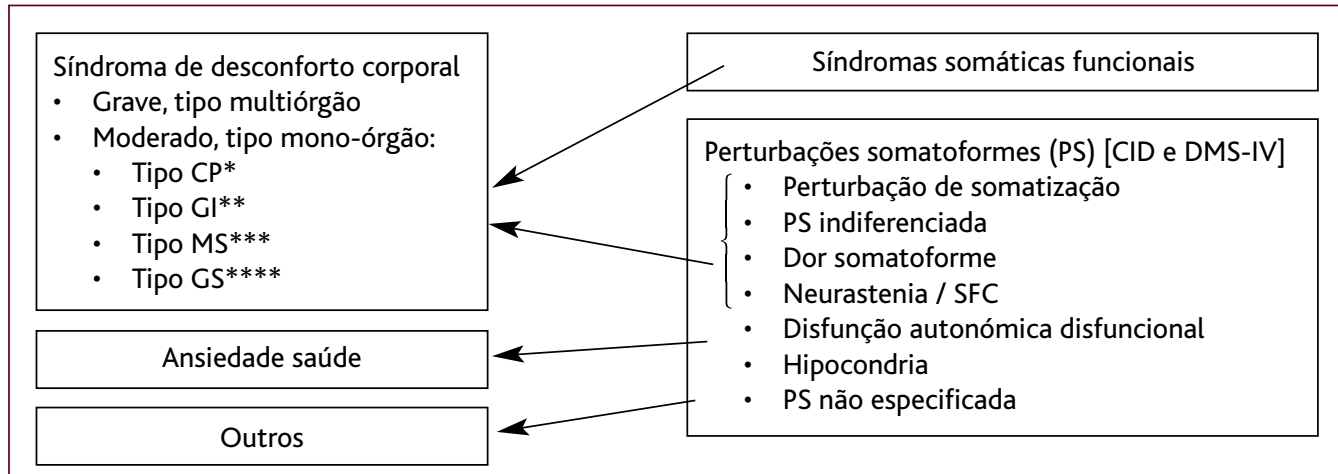

*) 3 ou mais sintomas cardiopulmonares (CP): palpitações, peso no peito, desconforto precordial, dispneia sem esforço, hiperventilação, suores quentes ou frios, tremores ou arrepios, boca seca, impressão no estomago, rubor ou corar.

$\left.{ }^{* *}\right) 3$ ou mais sintomas gastrointestinais (GI): dores abdominais, peristaltismo frequente com fezes moles, sentir-se inchado/cheio de gases/distendido, regurgitações, obstipação, diarreia, náuseas, vómitos, sensação de queimadura no peito ou epigastro.

***) 3 ou mais sintomas músculo-esqueléticos (MS): dores dos braços ou pernas, dores musculares, dores articulares, sensação de paresia ou fraqueza localizada, dores nas costas, dor migratória de um local para o outro, entorpecimento desagradável, sensação de formigueiro.

${ }^{* * * *}$ ) 3 ou mais sintomas gerais: dificuldades de concentração, perturbação da memória, fadiga excessiva, cefaleias, tonturas.

SDC multiórgão: mais de 3 sintomas de 3 ou 4 dos grupos de sintomas antes referidos.

SDC mono-órgão: 3 ou mais sintomas de 1 ou 2 dos sintomas dos grupos descritos ou mais de 4 sintomas da lista anterior.

da e recorrendo a

métodos de análise fatorial. Analisando 62 sintomas, referidos por pelo menos 10 doentes, e usando a análise fatorial de componentes principais, emergiu um modelo de três agrupamentos de sintomas: cardiopulmonar (CP), gastrointestinal (GI) e músculo-esquelético (MS). Para além destes, consideraram um quarto grupo de sintomas gerais (GS) formado por cinco sintomas inespecíficos (cefaleias, tonturas, alterações da memória, dificuldades de concentração e fadiga). Por outro lado, ao estudarem os doentes com a análise de clusters usando classes latentes verificaram que os grupos de sintomas têm elevada probabilidade de estarem presentes nos mesmos doentes, sugerindo que eles são a manifestação de um fenómeno comum latente. Para além disso, classificaram os doentes em três classes de gravidade: sem desconforto corporal (nonbodily distress), moderado desconforto corporal (modest bodily distress) e grave desconforto corporal (severe bodily stress). Embora os sintomas funcionais sejam um continuum, desde um mínimo até muitos sintomas, os autores decidiram considerar a presença de três sintomas como o ponto de corte e consideraram dois tipos de SDC: um mono-órgão, moderado, e outro multiórgãos, grave. Por sua vez, o SDC moderado ou mono-órgão tem quatro subtipos, já definidos: CP, GI, MS e GS. Já o SDC grave ou multiórgãos caracteriza-se por mais de quatro sintomas de qualquer um dos grupos. Estes clusters de sintomas foram confirmados numa população de 2.480 pacientes dos cuidados de saúde primários. ${ }^{22}$

Uma síndroma pressupõe a crença de que na base dos seus sintomas está um fenómeno latente comum. Os autores não encontraram evidência de que os grupos de sintomas sejam entidades clinicamente independentes atribuíveis a uma alteração específica de órgão porque eles estavam significativamente interrelacionados e já outros autores tinham demonstrado que a comorbilidade de síndromas funcionais é muito frequente. ${ }^{23-24}$ Assim, concluem que as síndromas funcionais identificadas correspondem ao mesmo fenómeno latente comum. A este fenómeno chamaram de desconforto corporal (bodily distress), porque o perfil de sintomas indica ser mais devido a mecanismos controlados centralmente do que a alterações dos sistemas orgânicos específicos periféricos. 
Utilizando a mesma amostra de doentes e aplicando a Schedules for Clinical Assessment in Neuropsychiatry, os autores verificaram que a SDC incluía todas as síndromas de fibromialgia, de fadiga crónica, de hiperventilação, 98\% do cólon irritável e, pelo menos, $90 \%$ de dores precordiais inespecíficas, de dor e qualquer perturbação somatoforme. Os perfis sintomáticos dos subtipos, mono-órgão, do SDC eram semelhantes às síndromas funcionais somáticas com um grau de concordância de 90 a 95\%. Concluem os autores que o diagnóstico de SDC cobre todo o leque de síndromas funcionais somáticas e de perturbações somatoformes, tendo o potencial de substituir um número considerável de rótulos diagnósticos até agora prevalentes nas síndromas funcionais somáticas (Figura 1). A designação de SDC tem a vantagem de oferecer uma linguagem e entendimento comuns para a compreensão das síndromas funcionais somáticas. ${ }^{25}$

Creed e colaboradores ${ }^{9}$ apontam dez princípios para que um termo seja considerado útil: 1) ser aceite pelos doentes; 2) ser aceite e usável pelos profissionais de saúde, sendo previsível a sua utilização na prática clínica; 3) não reforçar o pensamento dualístico inútil; 4) poder ser usado noutros doentes com outras patologias declaradas; 5) poder ser um diagnóstico por si; 6) ter um núcleo teórico claro; 7) facilitar a comunicação e tratamento multidisciplinar; 8) ter significados semelhantes em culturas diferentes; 9) ser neutro quanto à etiologia e patologia; 10) ter acrónimo aceitável. Segundo os mesmos autores, a designação de SDC parece obedecer a todos estes princípios.

O modelo metateórico de terapia cognitivo-comportamental ${ }^{26}$ baseia-se em algumas das teorias explicativas da SDC, como a da sensibilização, ${ }^{27}$ da sensibilidade, amplificação somatossensorial, da desregulação endócrina, ${ }^{28}$ dos comportamentos de dolência e do filtro sensorial. ${ }^{29}$ Existem outras teorias explicativas para os sintomas somatoformes que podem ser revistas no estudo de Olde Hartmann ${ }^{30}$ e de Van Ravenzwaaij e colaboradores. ${ }^{31}$ Este modelo enfatiza a natureza complexa destes sintomas, vincando a realidade da integração mente-corpo como um sistema único e indissociável. Para além disso, permite ao clínico melhorar a comunicação com o doente, fornece uma explicação plausível e tangível para os sintomas persistentes, possibilitando um diálogo terapêutico construtivo. Há, inclusive, propostas de taxonomia para as explicações a dar aos doentes. ${ }^{32}$

Neste contexto, o conceito de SDC parece-nos ser de utilidade clínica, sendo que os cuidados de saúde primários constituem o local de eleição para a abordagem destes «doentes», sem prejuízo de recurso episódico a outras especialidades, nomeadamente à psiquiatria. No entanto, pensamos que o SDC não deve ser entendido como entidade nosológica per si, mas antes como um conceito operacional. Ele designa uma situação clínica que exige uma abordagem específica, devendo ser vista como explicação temporária, passível de mudar sempre que existam indícios de outra entidade nosológica, ou seja, o SDC deve ser visto como um «diagnóstico» datado.

Claro que ainda há um longo caminho de investigação a percorrer para a consolidação do SDC que, como salientamos, captura diferentes diagnósticos dispersos por diferentes capítulos da CID-10 e as perturbações somatoformes da DSM-IV, entretanto ultrapassada pela DSM-V. Mas tem as vantagens de ser bem aceite pelos médicos dos cuidados de saúde primários ${ }^{21} \mathrm{e}$ de nascer de estudos empíricos realizados neste nível de cuidados, embora lhe escapem os sintomas isolados, mas incapacitantes. ${ }^{33}$ Finalmente, fica o desafio de estudar a sua aplicabilidade em Portugal, tendo em atenção o contexto cultural e linguístico.

\section{REFERÊNCIAS BIBLIOGRÁFICAS}

1. Mumford DB, Devereux TA, Maddy PJ, Johnston JV. Factors leading to the reporting of 'functional' somatic symptoms by general practice attenders. Br J Gen Pract. 1991;41(352):454-8.

2. Peveler R, Kilkenny L, Kinmonth AL. Medically unexplained physical symptoms in primary care: a comparison of self-report screening questionnaires and clinical opinion. J Psychosom Res. 1997;42(3):245-52.

3. Reid S, Wessely S, Crayford T, Hotopf M. Medically unexplained symptoms in frequent attenders of secondary health care: retrospective cohort study. BMJ. 2001;322(7289):767

4. Li CT, Chou YH, Yang KC, Yang CH, Lee YC, SuTP. Medically unexplained symptoms and somatoform disorders: diagnostic challenges to psychiatrists. J Chin Med Assoc. 2009;72(5):251-6.

5. Nunes JM, Yaphe J, Santos I. Sintomas somatoformes em medicina de família: um estudo descritivo da incidência e evolução em uma unidade de saúde familiar de Portugal [Somatoform symptoms in family medicine: a descriptive study of incidence and outcome in a family health center in Portugal]. Rev Bras Med Fam Comunidade. 2013; 8(28):164-71. Portuguese

6. Sousa JC. Ups! Será que é MUPS? [Oops! Is it MUPS?] [Editorial]. Rev Port Clin Geral. 2006;22(3):277-9. Portuguese.

7. Nunes JM. Carta ao director da RPCG (sobre MUPS) [Letter to the editor]. Rev Port Clin Geral. 2006;22(4):513-5. Portuguese

8. Mayou R, Farmer A. Functional somatic symptoms and syndromes. BMJ. 2002;325:265-8.

9. Creed F, Guthrie E, Fink P, Henningsen P, Rief W, Sharpe M, et al. Is there a better term than 'medically unexplained symptoms'? J Psychosom Res. 2010;68(1):5-8.

10. American Psychiatric Association. DSM-IV-TR: manual de diagnóstico e estatística das perturbações mentais. $4^{\text {a }}$ ed. Lisboa: Climepsi Editores; 2004. ISBN 9789727960200

11. World Health Organization. ICD-10: international statistical classification of disease related health problems [Internet]. 10th revision. Geneva:WHO; 2016. Available from: http://apps.who.int/classifications/icd10/browse/2016/en 
12. American Psychiatric Association. What is somatic symptom disorders [Internet]. Arlington, VA: APA; 2010 [cited 2011 May 15]. Available from: https://www.psychiatry.org/patients-families/somatic-symptom-disorder/what-is-somatic-symptom-disorder

13. American Psychiatric Association. J 00 Complex somatic disorder [Internet]. Arlington, VA: APA; 2010 [cited 2011 May 15]. Available from: http://www.dsm5.org

14. Smith RC, Gardiner JC, Lyles JS, Sirbu C, Dwamena FC, Hodges A, et al. Exploration of DSM-IV criteria in primary care patients with medically unexplained symptoms. Psychosom Med. 2005;67(1):123-9.

15. Al Busaidi ZQ. The concept of somatisation: a cross-cultural perspective. Sultan Qaboos Univ Med J. 2010;10(2):180-6.

16. Fink $P$, Rosendal $M$, Olesen F. Classification of somatization and functional somatic symptoms in primary care. Aust N Z J Psychiatry. 2005;39(9):772-81.

17. Escobar Jl, Hoyos-Nervi C, Gara M. Medically unexplained physical symptoms in medical practice: a psychiatric perspective. Environ Health Perspect. 2002;110 Suppl 4:631-6.

18. Lucini D, Pagani M. From stress to functional syndromes: an internist's point of view. Eur J Intern Med. 2012;23(4):295-301.

19. Engel CC Jr, Katon WJ. Population and need-based prevention of unexplained physical symptoms in the community. In: Joellenbeck LM, Russell PK, Guze SB, editors. Strategies to protect the health of deployed U.S. forces: medical surveillance, record keeping, and risk reduction. Washington DC: National Academy Press; 1999. p. 173-212. ISBN 9780309172523

20. Fink P, Toft T, Hansen MS, Ørnbøl E, Olesen F. Symptoms and syndromes of bodily distress: an exploratory study of 978 internal medical, neurological, and primary care patients. Psychosom Med. 2007;69(1):30-9.

21. Goldberg DP, Lam TP, Minhas F, Razzaque B, Robles R, Bobes J, et al. Primary care physicians' use of the proposed classification of common mental disorders for ICD-11. Fam Pract. 2017 May 4. [Epub ahead of print]

22. Budtz-Lilly A, Fink P, Ørnbøl E, Vestergaard M, Moth G, Christensen KS, et al. A new questionnaire to identify bodily distress in primary care: the 'BDS checklist'. J Psychosom Res. 2015;78(6):536-45.

23. Wessely S, Nimnuan C, Sharpe M. Functional somatic syndromes: one or many? Lancet. 1999;354(9182):936-9.

24. Whitehead WE, Winget C, Fedoravicius AS, Wooley S, Blackwell B. Learned illness behavior in patients with irritable bowel syndrome and peptic ulcer. Dig Dis Sci. 1982;27(3):202-8.
25. Fink P, Schröder A. One single diagnosis, bodily distress syndrome, succeeded to capture 10 diagnostic categories of functional somatic syndromes and somatoform disorders. J Psychosom Res. 2010;68(5):415-26.

26. Deary V, Chalder T, Sharpe M. The cognitive behavioural model of medically unexplained symptoms: a theoretical and empirical review. Clin Psychol Rev. 2007;27(7):781-97.

27. Rief W, Barsky AJ. Psychobiological perspectives on somatoform disorders. Psychoneuroendocrinology. 2005;30(10):996-1002.

28. Lam TP, Goldberg DP, Dowell AC, Fortes S, Mbatia JK, Minhas FA, et al. Proposed new diagnoses of anxious depression and bodily stress syndrome in ICD11-PHC: an international focus group study. Fam Pract. 2013;30(1):76-87.

29. Rief W, Broadbent E. Explaining medically unexplained symptoms-models and mechanisms. Clin Psychol Rev. 2007;27(7):821-41.

30. Olde Hartman TC. Medically unexplained symptoms: a qualitative analysis of the literature. In: Olde Hartman TC. Persistent medically unexplained symptoms in primary care: the patient, the doctor and the consultation [Dissertation]. Nijmegen: Radboud University; 2011. p. 165-78. Available from: http://repository.ubn.ru.nl/bitstream/handle/2066/91314/91314.pdf?sequence $=1$

31. Van Ravenzwaaij J, Olde Hartman TC, Van Ravesteijn H, Eveleigh R, Van Rijswijk E, Lucassen PL. Explanatory models of medically unexplained symptoms: a qualitative analysis of the literature. Ment Health Fam Med. 2010;7(4):223-31.

32. Morton L, Elliott A, Cleland J, Deary V, Burton C. A taxonomy of explanations in a general practitioner clinic for patients with persistent 'medically unexplained' physical symptoms. Patient Educ Couns. 2017;100(2):224-30.

33. Budtz-Lilly A, Schröder A, Rask MT, Fink P, Vestergaard M, Rosendal M. Bodily distress syndrome: a new diagnosis for functional disorders in primary care? BMC Fam Pract. 2015;16:180.

\section{CONFLITO DE INTERESSES}

O autor declara não ter conflitos de interesses.

\section{ENDEREÇO PARA CORRESPONDÊNCIA}

E-mail: josemendesnunes@gmail.com

Recebido em 19-04-2016

Aceite para publicação em 27-07-2017

\begin{abstract}
BODILY DISTRESS SYNDROME: REUNITING WHAT OTHER SPECIALITIES HAVE DIVIDED

The author defends the concept of the bodily distress syndrome (BDS) as a unifying entity of somatic syndromes and somatoform symptoms. These patients have physiological, cognitive, and behavioural characteristics that require a comprehensive approach. We require the integration of a number of pseudo-diagnoses that are side effects of medical specialization. Patients most in need of a holistic view are paradoxically disintegrated by the approach of many medical specialties. They simply add diagnostic labels that reduce the patient's perception of health. The concept of BDS accepts the complexity of these symptoms. It rejects the mind-body duality and the negative definition of the condition as the absence of a medical explanation. Because this approach depends on the doctorpatient relationship and continuity of care, the family physician is primarily responsible for addressing the needs of these patients, with rational use of referral to other specialties.
\end{abstract}

Keywords: Functional somatic syndromes; Somatoform disorders; Bodily distress syndrome 